\title{
Protocolo de abordagem de derrame pleural
}

\section{Pleural effusion management protocol}

\author{
George Cavalcante Dantas ${ }^{1}$. Ricardo Coelho Reis². \\ 1 Especialista em Clínica Médica, Residente de Pneumologia pelo Hospital de Messejana, Fortaleza, Ceará, Brasil. 2 Mestre em \\ Medicina Clínica, Pneumologista e Intensivista. Pneumologista do Hospital Universitário Walter Cantídio (HUWC), Universidade \\ Federal do Ceará (UFC), Fortaleza, Ceará, Brasil.
}

\section{RESUMO}

O derrame pleural (DP) é uma patologia médica comum, com incidência aproximada de 1 milhão de casos ao ano nos Estados Unidos. Estima-se que a incidência no Brasil seja semelhante, tornando-se de grande importância na prática clínica. O derrame pleural ocorre quando existe um acúmulo de líquido entre a pleura visceral e parietal. $\mathrm{O}$ transudato geralmente é devido a alterações no controle do fluido corporal, enquanto o exsudato é frequentemente causado por processo inflamatório ou maligno. Neste protocolo, é abordado inicialmente a fisiopatologia, os sinais, os sintomas e as principais causas de DP, incluindo os de etiologia indeterminada. Em seguida, é explorada a investigação inicial, passando por métodos diagnósticos com complexidade crescente, com o objetivo de estabelecer um diagnóstico rápido, reduzindo técnicas invasivas e desnecessárias. Este protocolo apresenta um fluxograma para investigação geral do DP e a abordagem do tratamento de suas principais etiologias, facilitando e orientando a conduta médica no Hospital Universitário Walter Cantídio (HUWC).

Palavras-chave: Derrame pleural. Diagnóstico. Protocolo. Fluxograma.

\section{ABSTRACT}

Pleural effusion (PE) is a common medical condition, affecting approximately 1 million people each year in the United States. It is estimated that the incidence in Brazil is similar, resulting in a great importance in clinical practice. Pleural effusion occurs when there is an accumulation of fluid between the visceral and parietal pleura. The transudate is usually due to a distortion in body fluid mechanics, while the exudate is often caused by inflammatory or malignant process. In this protocol, it is initially approached the pathophysiology, signs, symptoms and major causes of PE, including those of undetermined etiology. Then, it goes on with the initial investigation, through diagnostic methods with increasing complexity, in order to establish an early diagnosis, reducing invasive and unnecessary procedures. This protocol provides a flowchart for general investigation of the EP and the treatment of its main causes, facilitating and guiding the medical management at the Hospital Universitário Walter Cantídio (HUWC).

Keywords: Pleural effusion. Diagnostic. Protocol. Flowchart.

Autor correspondente: George Cavalcante Dantas, Rua Carlos Vasconcelos 808, Meireles, Fortaleza, Ceará, Brasil. Telefone: +55 85 $98610-9844$. E-mail: georgedantas@gmail.com

Conflito de interesses: Não há qualquer conflito de interesses por parte de qualquer um dos autores.

Recebido em: 05 Jul 2017; Revisado em: 28 Jul 2017; Aceito em: 06 Ago 2017. 


\section{INTRODUÇÃO}

Embora no Brasil não existam dados concretos da incidência de derrames pleurais (DP), estima-se que esta seja semelhante à dos Estados Unidos, com cerca de 1 milhão de pacientes ao ano. ${ }^{1}$

A pleura é formada por duas membranas serosas, uma que recobre o pulmão, chamada de pleura visceral, e outra que recobre a face interna da parede torácica, chamada de pleura parietal. O espaço pleural é um espaço virtual delimitado pelas duas pleuras e que contém uma pequena quantidade de líquido $(0,1 \mathrm{ml} / \mathrm{kg})$ com a função de lubrificação, facilitando o deslizamento pleural. ${ }^{1,2}$

Os derrames pleurais podem ou não estar associados à doença da pleura. Em geral, os causados por doenças da pleura em si assemelham-se ao plasma (exsudatos), enquanto os que ocorrem com uma pleura normal são ultrafiltrados do plasma (transudatos). As pressões oncóticas e hidrostáticas regulam o movimento do líquido entre a pleura. Um desequilíbrio desta relação pode causar acúmulo anormal de transudato. Processos inflamatórios ou malignos, por sua vez, podem promover aumento da permeabilidade da membrana capilar e pleural ou bloqueio linfático, o que permite o acúmulo de exsudato. ${ }^{1,2}$

\section{AVALIAÇÃO INICIAL}

A anamnese e o exame físico são fundamentais para uma avaliação de derrame pleural. Dados da vida profissional, do uso de medicamentos, conforme Tabela 1, e da história médica clínica e cirúrgica podem ajudar na elaboração de uma suspeita etiológica, exemplificados na Tabela 2.1,3

Tabela 1. Principais medicamentos associados a derrame pleural

\begin{tabular}{l}
\hline Medicamentos \\
\hline Metotrexato \\
Amiodarona \\
Fenitoína \\
Nitrofurantoína \\
Beta-bloqueadores \\
\hline
\end{tabular}

Adaptado de: Hooper C, Lee YC, Maskell N; BTS Pleural Guideline Group. ${ }^{3}$

Tabela 2. Anamnese e possíveis etiologias associadas.

\begin{tabular}{ll}
\hline ANAMNESE & POSSíVEL ETIOLOGIA \\
\hline Exposição ao asbesto (amianto) & Derrame benigno pelo asbesto, mesotelioma \\
Contactante de tuberculose & Tuberculose \\
Antecedente de etilismo ou esteatorreia & Pancreatite, pseudocisto pancreático \\
Manipulação cirúrgica abdominal & Pancreatite, abscesso subfrênico \\
Antecedente de neoplasia & Metástase \\
Insuficiência cardíaca congestiva, insuficiência renal ou hepática & Transudato \\
IAM & Síndrome de Dressler \\
Fatores de risco para TVP & Embolia pulmonar \\
\hline
\end{tabular}

IAM: Infarto Agudo do Miocárdio; TVP: Trombose Venosa Profunda.

Adaptado de: Pereira CA, Holanda MA. ${ }^{1}$

O exame do tórax revela percussão maciça, frêmito ausente e diminuição ou ausência de murmúrio vesicular. Turgência jugular, S3 galopante ou edema periférico sugerem insuficiência cardíaca congestiva; e ictus de ventrículo direito (VD) propulsivo ou TVP sugerem tromboembolismo pulmonar. A presença de linfadenopatia ou hepatoesplenomegalia levanta a hipótese de neoplasias; e ascite pode sugerir uma causa hepática. ${ }^{4}$

Os sintomas mais frequentes de DP são dor, tosse e dispneia. A "dor pleurítca" geralmente se manifesta com moderada intensidade, em pontada e variando com os movimentos respiratórios. Nos processos infecciosos, como na pneumonia, esta costuma ser aguda e crescente. Já nos processos embólicos e no pneumotórax apresenta-se de forma súbita. A inervação tanto do gradil costal quanto das pleuras diafragmática e mediastínica pode transferir a manifestação da dor para longe do local real. A tosse geralmente é seca, podendo vir em acessos, consequência da estimulação das terminações nervosas no processo inflamatório. A presença de dispneia é bastante variável, podendo apresentar-se de forma súbita ou crescente. Em geral, o grau é proporcional à quantidade de líquido e à velocidade de acúmulo. ${ }^{1}$

\section{AVALIAÇÃO INICIAL POR IMAGEM}

Os líquidos pleurais se acumulam na parte inferior da cavidade torácica devido aos pulmões, que apresentam menor densidade que o líquido. Na radiografia de tórax póstero-anterior (PA), é necessária uma quantidade de líquido maior que $200 \mathrm{ml}$ para obliterar o recesso costofrênico lateral, enquanto na radiografia em perfil, basta $50 \mathrm{ml}$ para alterar o recesso costofrênico posterior. Em terapia intensiva, a maioria das radiografias é anteroposterior (AP) em decúbito dorsal. Neste caso, observase um aumento da densidade no hemitórax, com preservação da trama vascular. Derrame subpneumônico ocorre quando o líquido se encontra entre a superfície do diafragma e o pulmão, 
podendo ser de difícil visualização na radiografia em PA. Uma radiografia em decúbito lateral pode ser realizada para estimar a quantidade de líquido e na suspeita de derrame loculado. ${ }^{2,3}$

A ultrassonografia (USG) está indicada nos casos de dúvida e para ajudar na localização da punção nos derrames pleurais pequenos ou loculados. Muitos trabalhos mostram sucesso na punção guiada por USG em até $88 \%$ nos pacientes que falharam na primeira tentativa. Ela pode detectar septações no espaço pleural com sensibilidade maior que a tomografia.,

\section{INDICAÇÃO DE TORACONCETESE}

A indicação de toracocentese diagnóstica ocorre na presença de derrame pleural maior que $10 \mathrm{~mm}$ na radiografia de decúbito lateral ou na USG, sem causa conhecida. Entretanto, se houver segurança no diagnóstico clínico, esta pode não ser necessária. Se o paciente for portador de insuficiência cardíaca congestiva, com derrame bilateral de tamanhos semelhantes, afebril e sem dor torácica, um tratamento com diurético pode ser tentado. Toracocentese está indicada se houver derrame unilateral ou sem melhora após 48 horas do início de diuréticos. ${ }^{4}$

A toracocentese inicial é geralmente realizada com propósito diagnóstico, a menos que o paciente apresente dispneia ao repouso, onde a forma terapêutica é realizada com retirada de até $1500 \mathrm{ml}$. A realização guiada por USG está indicada na presença de alguma dificuldade em obter o líquido pleural ou DP de pequeno tamanho. Não é necessário radiografia de controle de rotina, somente em casos de ar durante punção, aparecimento de tosse, dor torácica ou dispneia. ${ }^{4}$

Não existe contraindicação absoluta ao procedimento. Em algumas situações, deve haver uma maior cautela, tais como: Tempo de Ativação da Protrombina (TAP) ou Tempo de Tromboplastina Parcial Ativada (TTPA) maior que duas vezes a normalidade; quantidade de plaquetas menor que $50.000 / \mathrm{mm}^{3}$; pacientes em ventilação mecânica; infecção ativa no local da punção. $^{6}$

\section{ANÁLISE DO LÍQUIDO PLEURAL}

\section{Aparência do líquido}

A aparência do líquido pode promover dicas diagnósticas, tais como: aspecto sanguinolento sugere malignidade, tromboembolismo pulmonar ou trauma; líquido leitoso pode indicar quilotórax ou pseudoquilotórax; aspecto purulento define empiema; odor pútrido pode indicar infecção por anaeróbios; e odor de urina sugere provavelmente urinotórax. ${ }^{4}$

\section{Diferenciação entre exsudato e transudato}

O primeiro passo diante da investigação é determinar se o líquido é um exsudato ou transudato, podendo guiar as causas do mesmo, conforme Tabela $3 .^{3} \mathrm{O}$ método mais difundido e aceito é o critério de Light. Este possui sensibilidade de 98\% para diagnóstico de exsudato e consegue diferenciar exsudato de transudato em 93 a 96\% dos casos. Consiste em três parâmetros, bastando um positivo para caracterizar como exsudato: proteína pleural/sérica $>0,5 ;$ LDH pleural/sérico $>0,6 ; \mathrm{LDH}>2 / 3$ do limite superior da normalidade para nível sérico. $\mathrm{O}$ ponto de corte dos parâmetros é útil para aumentar a sensibilidade para exsudato na avaliação de um derrame pleural. ${ }^{1,4}$

O critério de Light tem acurácia menor nos transudatos causados por insuficiência cardíaca em pacientes que utilizam diuréticos por mais de 48 horas. Nestes casos, recomenda-se a medida do gradiente de albumina. Para confirmação de transudato, o valor de albumina sérica menos albumina pleural deve ser maior que $1,2 \mathrm{~g} / \mathrm{dl}$. Este critério não pode ser usado isoladamente, pois classifica $13 \%$ dos exsudatos como transudatos. ${ }^{1,4}$ Proteína pleural maior que $2,9 \mathrm{~g} / \mathrm{dl}$ e colesterol pleural maior que $43 \mathrm{mg} / \mathrm{dl}$ também sugerem tratar-se de exsudato, conforme Tabela 4.,7

Tabela 3. Causas de derrame pleural.

\begin{tabular}{lc}
\hline TRANSUDATO & EXSUDATO \\
\hline Falência ventricular esquerda & Causas comuns \\
Cirrose hepática & Malignidade \\
& Derrame parapneumônico \\
& Tuberculose \\
Hipoalbuminemia & Causas pouco comuns \\
Hipotireoidismo & Embolismo Pulmonar \\
Síndrome nefrótica & Artrite reumatoide/auto-imune \\
Estenose mitral & Pancreatite \\
& Pós-IAM/pós-revascularização do miocárdio \\
Pericardite constrictiva & Causas raras \\
Urinotórax & Síndrome da unha amarela \\
Síndrome de Meigs & Medicamentos \\
\hline
\end{tabular}

Adaptado de: Hooper C, Lee YC, Maskell N; BTS Pleural Guideline Group. ${ }^{3}$ 
Tabela 4. Sensibilidade e especificidade para exsudato.

\begin{tabular}{lcc}
\hline TESTES & SENSIBILIDADE & ESPECIFICIDADE \\
\hline Critério de Light & $98 \%$ & $83 \%$ \\
Colesterol pleural $>60 \mathrm{mg} / \mathrm{dl}$ & $54 \%$ & $92 \%$ \\
Colesterol pleural $>43 \mathrm{mg} / \mathrm{dl}$ & $75 \%$ & $80 \%$ \\
Relação colesterol pleural sobre colesterol sérico $>0,3$ & $89 \%$ & $81 \%$ \\
Gradiente albumina soro-pleural & $87 \%$ & $92 \%$ \\
\hline
\end{tabular}

Adaptado de: Light RW. ${ }^{4}$

Na suspeita de transudato, pode-se coletar uma amostra de soro e líquido pleural somente para LDH e proteína. Se o paciente utiliza diurético, é necessário acrescentar a dosagem pleural e sérica de albumina. Na suspeita de exsudato, a dosagem de outros parâmetros é fundamental para avaliação diagnóstica. Os principais são: $\mathrm{pH}$, glicose, esfregaço e culturas de organismos, adenosina deaminase (ADA) e contagem celular total e diferencial. ${ }^{1}$

\section{Celularidade total e diferencial}

O predomínio de neutrófilos no líquido pleural (> $50 \%$ das células) indica processo agudo. Ocorrem em derrame parapneumônico, tromboembolismo pulmonar e tuberculose (TB) aguda. ${ }^{4} \mathrm{Se}$ a celularidade evidenciar predomínio de linfócitos ( $>50 \%$ das células), as principais causas são malignidade e TB. Uma proporção muito alta de linfócitos (> 80\%) sugere TB, linfoma, derrame reumatoide crônico, sarcoidose e pós-revascularização do miocárdio. Derrame eosinofílico é definido como contagem de eosinófilos $\geq 10 \%$ das células. As causas mais comuns são a presença de ar ou sangue no espaço pleural. É um achado relativamente inespecífico, podendo ser encontrado também em derrames parapneumônicos, síndrome de Churg-Strauss, linfoma, infarto pulmonar, parasitoses, derrames por medicamentos e malignidade. ${ }^{3}$ Células mesoteliais podem ser encontradas em líquidos pleurais normais ou em transudatos. Um dado clínico relevante é que nos exsudatos por TB essas células são infrequentes com percentual $<5 \%$. ${ }^{7}$

\section{pH pleural}

O líquido pleural possui aproximadamente $\mathrm{pH}$ de 7,60. Transudatos geralmente apresentam $\mathrm{pH}$ entre 7,40 e 7,55, enquanto a maioria dos exsudatos possuem $\mathrm{pH}$ entre 7,30 e 7,45. ${ }^{7}$ Uma acidose do líquido pleural $(\mathrm{pH}<7,30)$ ocorre em malignidade, derrame infeccioso complicado, doenças do tecido conjuntivo (particularmente artrite reumatoide), TB e ruptura esofágica. Em derrames malignos, um baixo pH está associado à uma menor sobrevida, doença mais avançada e menor taxa de sucesso de pleurodese. Na prática clínica, o uso mais importante do $\mathrm{pH}$ pleural é na decisão de tratar infecção pleural com drenagem torácica. Um $\mathrm{pH}<$ 7,20 é o discriminador mais específico para infecção pleural complicada. ${ }^{3,5}$

\section{Glicose}

A presença de glicose pleural $<60 \mathrm{mg} / \mathrm{dl}$ indica um derrame parapneumônico complicado ou maligno. As menores concentrações de glicose são vistas na artrite reumatoide e empiema, sendo algumas vezes até indetectáveis. Outras causas são TB, lúpus e ruptura esofágica. ${ }^{3,7}$

\section{Adenosina deaminase (ADA)}

A ADA é uma enzima que tem função importante na proliferação e na diferenciação dos linfócitos e na maturação dos monócitos. ${ }^{1}$ Pode ajudar no diagnóstico diferencial entre causa maligna e TB em um derrame linfocítico, mas com esfregaço e cultura negativa. ${ }^{7} \mathrm{O}$ valor de corte usado para TB é > 40 U/1. ${ }^{1,4}$ Pode ser encontrada elevada também no empiema e artrite reumatoide. Restringindo o uso apenas em derrames linfocíticos e dosando apenas a isoenzima ADA-2 pode-se reduzir significativamente os falsos positivos. ${ }^{3}$

\section{Colesterol e triglicerídeos}

Se o líquido pleural apresenta aspecto leitoso, a suspeita de quilotórax e pseudoquilotórax deve ser aventada. Neste caso, valores de triglicerídeos $>110 \mathrm{mg} / \mathrm{dl}$ e presença de quilomícrons indicam quilotórax; valores de triglicerídeos $<50$ $\mathrm{mg} / \mathrm{dl}$ e colesterol $>200 \mathrm{mg} / \mathrm{dl}$ indicam peseudoquilotórax. ${ }^{1,3}$ As causas mais frequentes de quilotórax e pseudoquilotórax encontram-se na Tabela 5. ${ }^{3}$ Ocasionalmente, um empiema pode ser confundido com o quilotórax. Eles podem ser diferenciados com centrifugação, que deixa um sobrenadante límpido em casos de empiema, enquanto o quilo permanece leitoso. ${ }^{3}$

Tabela 5. Causas comuns de quilotórax e pseudoquilotórax.

\begin{tabular}{ll}
\hline QUILOTÓRAX & PSEUDOQUILOTÓRAX \\
\hline Trauma e cirurgia torácica & Tuberculose \\
Linfoma ou carcinoma metastático & Artrite reumatoide \\
$\begin{array}{l}\text { Desordem de linfáticos, TB, } \\
\text { cirrose, obstrução de veias centrais, } \\
\text { quiloascite }\end{array}$ & \\
Idiopático (10\%) & \\
\hline
\end{tabular}

Adaptado de: Hooper C, Lee YC, Maskell N; BTS Pleural Guideline Group. ${ }^{3}$ 


\section{Amilase}

Apesar de não medida rotineiramente, amilase pode ajudar no diagnóstico de possível etiologia pancreática ou esofágica. Níveis elevados de amilase pleural (maior que o limite superior da normalidade) ou relação entre amilase pleural e sérica maior que 1 podem indicar derrame pleural devido a pancreatite aguda, pseudocisto pancreático, ruptura do esôfago, malignidade e gravidez ectópica. ${ }^{1,3}$

\section{Testes para câncer}

Na suspeita de malignidade, citologia é um exame rápido e minimamente invasivo com sensibilidade de cerca de $70 \%$, dependendo da etiologia. ${ }^{4} \mathrm{~A}$ taxa de diagnóstico é maior para adenocarcinoma do que para mesotelioma, carcinoma de células escamosas, linfoma e sarcoma., Apesar das divergências na literatura, sugere-se que seja coletado cerca de $60 \mathrm{ml}$ de líquido pleural para enviar para estudo citológico. Se houver suspeita de linfoma, citometria de fluxo pode mostrar presença de célula clonal. ${ }^{3}$ No diagnóstico de câncer pleural, a biópsia às cegas acrescenta muito pouco à citologia. Dessa forma, a toracoscopia é o procedimento de escolha nos pacientes com suspeita de câncer com citologia negativa e sem imagem pulmonar evidente em tomografia computadorizada, com acurácia de aproximadamente 95\%. ${ }^{1,8}$ Marcadores tumorais não possuem acurácia significativa para uso rotineiro na avaliação de derrame pleural. ${ }^{7}$

\section{Bacterioscopia e culturas}

A bacterioscopia e a cultura do líquido pleural são importantes para diagnóstico e orientação da antibioticoterapia nos derrames pleurais infecciosos. A cultura para germes piogênicos é positiva em cerca de $60 \%$ dos casos. ${ }^{3}$ No entanto, no empiema, devido à intensa necrose, só é possível identificar o agente em menos de 20\% dos casos. Em casos de derrame tuberculoso, a baciloscopia é frequentemente negativa, com sensibilidade de no máximo 5\%. A cultura do líquido pleural em meio específico (Löwenstein Jensen) aumenta a sensibilidade para 10 a 35\%. ${ }^{1}$

\section{AVALIAÇÃO COMPLEMENTAR POR IMAGEM}

\section{Tomografia computadorizada}

A tomografia computadorizada (TC) detecta uma pequena quantidade de líquido pleural (menor que $10 \mathrm{ml}$ ). ${ }^{9}$ Deve ser realizada em derrames pleurais sem diagnóstico ou na presença de anomalias do parênquima pulmonar ou mediastino, ajudando no diagnóstico diferencial. ${ }^{5} \mathrm{O}$ líquido livre é visualizado como uma opacidade em foice na porção mais inferior da imagem. ${ }^{3} \mathrm{O}$ espessamento da pleura e as captações pós-contraste ("sinal da pleura dividida") sugere presença de inflamação, geralmente um exsudato. A TC consegue determinar a localização exata de massas pleurais, guiando com maior facilidade uma biópsia por agulha. ${ }^{9}$

\section{Ressonância nuclear magnética}

O acesso à ressonância nuclear magnética (RNM) ainda é limitado nos centros hospitalares, não fazendo parte da rotina no estudo do derrame pleural. Consegue distinguir com boa acurácia entre causas benignas e malignas de acordo com a diferença da intensidade de sinal de imagens pesadas em T2. Pode ser utilizada em pacientes onde o contraste ou a radiação estão contraindicados. ${ }^{3}$

\section{INVESTIGAÇÃO INVASIVA}

\section{Biópsia pleural percutânea}

A biópsia percutânea é útil em condições em que a pleura é acometida difusamente, como nos casos de TB pleural e granuloma não caseoso da artrite reumatoide, onde a sensibilidade pode chegar até $80 \%{ }^{8}$ Em regiões de alta incidência para TB, a biópsia percutânea às cegas apresenta um bom custo-benefício. ${ }^{3} \mathrm{Na}$ suspeita de malignidade, onde o acometimento pleural é salteado, a biópsia guiada por TC apresenta uma maior acurácia. ${ }^{8}$ As principais complicações do procedimento são: dor (1-15\%); pneumotórax (3-15\%); reação vasovagal (1-5\%); hemotórax $(<2 \%) .{ }^{3}$ Diferente da toracocentese, recomenda-se radiografia de tórax após a biópsia para descartar pneumotórax. ${ }^{5}$

\section{Toracoscopia com anestesia local e Toracoscopia assistida por vídeo}

Toracoscopia está indicada no estudo de exsudato, onde a toracocentese foi inconclusiva, mas a suspeita de malignidade é alta. Mediante introdução de um toracoscópio, visualiza-se a cavidade pleural, podendo obter amostras da pleura visceral e parietal de forma dirigida. A acurácia diagnóstica para neoplasia supera $90 \%$. Se indicado, uma pleurodese pode ser realizada no mesmo momento. ${ }^{5}$

A toracoscopia assistida por vídeo (VATS) é um procedimento realizado por cirurgiões torácicos e necessita de anestesia geral, não sendo a opção mais adequada em indivíduos frágeis ou com graves comorbidades. Uma vantagem da VATS em relação à toracoscopia com anestesia local é a possibilidade de o cirurgião realizar outras opções cirúrgicas, durante o procedimento. ${ }^{3}$

\section{Broncoscopia}

A broncoscopia não é uma técnica usada rotineiramente no estudo de derrame pleural. Deve ser aventada em casos de hemoptise ou sinais clínico-radiológicos de obstrução brônquica, com suspeita de massa pulmonar proximal. ${ }^{5}$ Deve ser realizada após drenagem torácica, para que seja realizada uma visualização adequada sem compressão extrínseca pelo líquido pleural. ${ }^{3}$

\section{DERRAME PLEURAL INDETERMINADO}

Após uma completa investigação, conforme Figura 1, incluindo biópsia por toracoscopia (acurácia diagnóstica de $96 \%$ ), um número significativo de pacientes com exsudato ainda permanece sem diagnóstico. A maioria dos casos de derrame indeterminado $(91,7 \%)$ apresenta um curso benigno, com resolução espontânea de $81,8 \%{ }^{3} \mathrm{O}$ acompanhamento é de fundamental importância nestes pacientes, com atenção ao diagnóstico de causas malignas ou tratáveis. A duração ideal do seguimento ainda não foi definida. ${ }^{8}$ 
Figura 1. Investigação de derrame pleural.

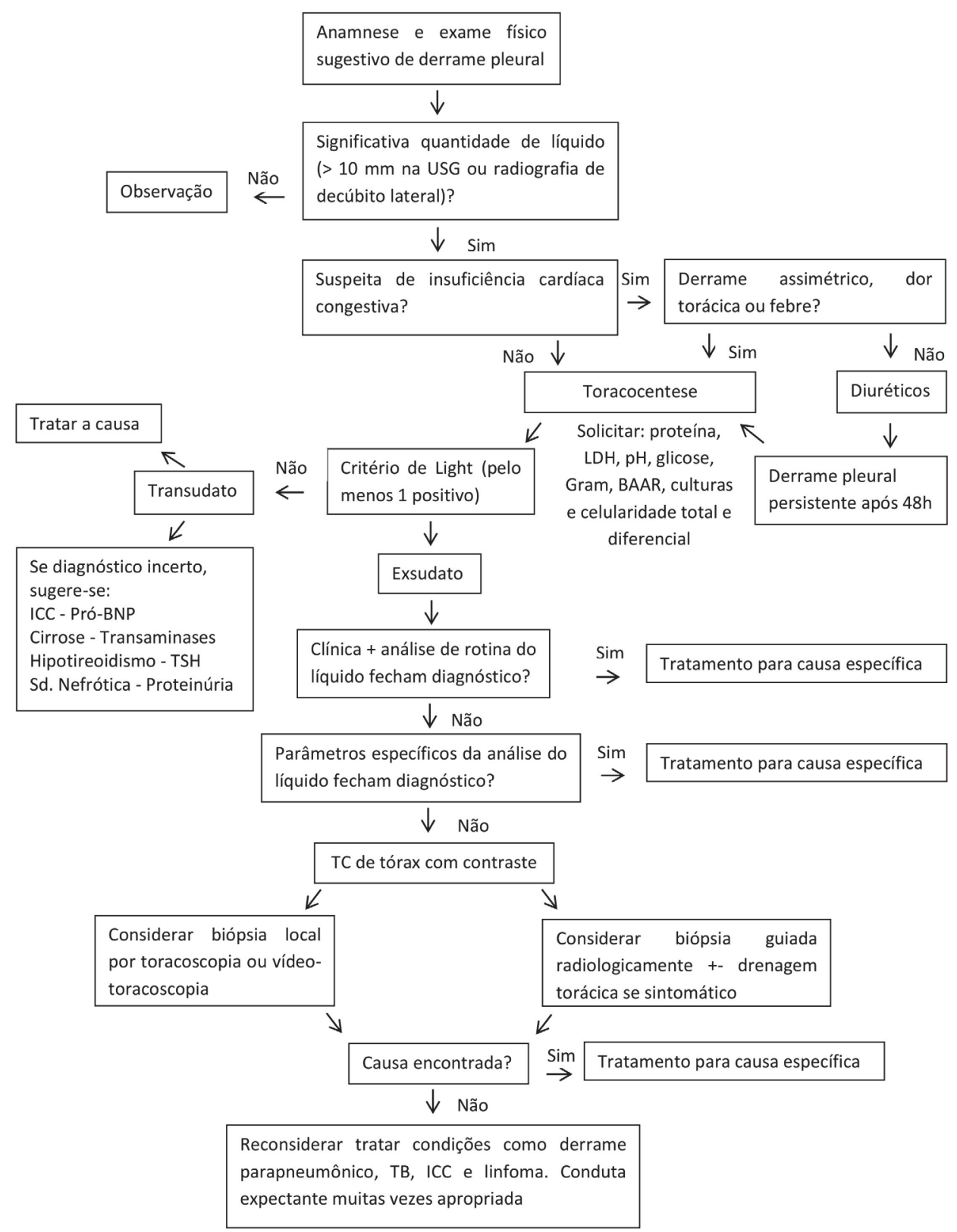

\section{TRATAMENTO DO DERRAME PLEURAL}

Neste tópico será abordado o tratamento geral das principais etiologias de derrame pleural e os casos recidivantes não neoplásicos.

\section{Derrame parapneumônico e empiema}

O tratamento do derrame parapneumônico segue uma sequência de eventos que vão desde uso de antibióticos até procedimentos cirúrgicos mais invasivos. A escolha do antibiótico ideal, na maioria das vezes, é empírica e fundamentada em dados epidemiológicos. Como regra geral, recomenda-se seguir as diretrizes brasileiras para pneumonia adquirida na comunidade em adultos imunocompetentes. Pequenos derrames $(<10 \mathrm{~mm})$ geralmente resolvem apenas com antibióticos. ${ }^{1}$

A toracocentese é a forma menos invasiva de abordagem. No entanto, deve ser realizada somente em derrames pequenos ou moderados (menos da metade do hemitórax) e que apresentem Gram e culturas negativas e $\mathrm{pH} \geq 7,2$, onde o risco de evolução desfavorável e de complicações é menor. A drenagem pleural, por sua vez, é indicada em DP parapneumônico de grande volume; Gram e culturas positivas; $\mathrm{pH}<7,2$; líquido pleural turvo ou francamente purulento; e em pacientes com evolução clínica inadequada com o uso exclusivo de antibióticos. Os parâmetros para retirada do dreno são a melhora clínica e radiológica e débido de drenagem $<100 \mathrm{ml} /$ dia para derrames não empiemáticos. No empiema, recomenda-se parada total da drenagem, seguida da confirmação por imagem (USG ou TC) da obliteração total do espaço pleural. ${ }^{1}$

A toracoscopia é preconizada quando a drenagem pleural foi ineficaz, necessitando de uma higiene mais eficaz da cavidade pleural. Sob visão direta é possível desfazer aderências e 
desencarceraropulmão. Se indicada precocemente, a toracoscopia pode evitar procedimentos mais invasivos. Para fins práticos, é aceito que pacientes com DP parapneumônico ou empiema sem melhora clínico-radiológica após 7 dias da antibioticoterapia e drenagem pleural sejam submetidos a tratamento cirúrgico. A decorticação aberta é um procedimento cada vez mais raro, devido à abordagem precoce com técnicas menos invasivas. A drenagem aberta, sem selo de água, está indicada nos casos de pacientes muito debilitados e com empiemas crônicos ( $>30$ dias de tratamento). Nos casos em que a cavidade residual é extensa, pode ser indicada ressecção de parte de um ou dois arcos costais, com formação de uma pleurostomia.

\section{Derrame pleural tuberculoso}

Em pacientes jovens, com quadro clínico e análise laboratorial do líquido pleural sugestiva, o tratamento empírico pode ser realizado após tentativa de se afastar outras causas de derrame pleural. O esquema terapêutico é igual ao das demais formas, com uso de 4 drogas combinadas rifampicina + isoniazida + pirazinamida + etambutol (RHZE) em um mesmo comprimido por 2 meses, seguido de 2 drogas rifampicina + isoniazida (RH) por mais 4 meses. ${ }^{1}$

Normalmente o DP tuberculoso evolui para cura sem sequelas ou com mínimo espessamento pleural. Recomenda-se o monitoramento clínico e radiológico da reabsorção do líquido após um a dois meses do início do tratamento. A manutenção do derrame pleural ou a presença de grandes espessamentos exigem pesquisa de diagnóstico diferencial e possível intervenção cirúrgica precoce. ${ }^{1}$

\section{Derrame pleural neoplásico}

Sendo o derrame pleural neoplásico uma manifestação de doença maligna avançada, o tratamento é considerado paliativo. ${ }^{1} \mathrm{~A}$ sobrevida média é em torno de 4 meses. ${ }^{10} \mathrm{O}$ objetivo é controlar de forma duradoura o derrame, aliviar os sintomas e reexpandir o pulmão. ${ }^{1}$ Derrame pleural assintomático não necessita de intervenção, já os que causam dispneia e não respondem ao tratamento do tumor de base requerem tratamento paliativo direcionado para cavidade pleural. ${ }^{10}$

Toracocentese de alívio é recomendada para derrames pleurais que reacumulam lentamente (mais de um mês), principalmente quando a sobrevida é curta ( $<3$ meses). ${ }^{10}$ A drenagem pleural não é recomendada isoladamente para controle definitivo do derrame maligno, já que a recidiva é elevada se não realizado pleurodose. ${ }^{1}$

A pleurodese consiste na abrasão ou instilação, na cavidade pleural, de substâncias esclerosantes, determinando uma

\section{REFERÊNCIAS}

1. Pereira CA, Holanda MA. Medicina respiratória. 1. ed. São Paulo: Ed. Atheneu; 2014.

2. Saguil A, Wyrick K, Hallgren J. Diagnostic approach to pleural effusion. Am Fam Physician. 2014;90(2):99-104.

3. Hooper C, Lee YC, Maskell N; BTS Pleural Guideline Group. intensa reação inflamatória aguda das superfícies pleurais. Isto resulta em fibrose e sínfise do espaço pleural, prevenindo a recidiva do DP. A pleurodese química é um dos principais procedimentos no caso de derrames recidivantes em pacientes com sobrevida maior que 3 meses. É contraindicada se não houver melhora da dispneia e reexpansão pulmonar de pelo menos $50 \%$ após toracocentese inicial ou drenagem. Dentre os agentes esclerosantes, o talco é um dos mais utilizados, com taxa de sucesso entre 70 e $90 \%$. Em suspensão, é aplicado ambulatorialmente através de um dreno de tórax. Já por aerossol ou polvilhamento, requer anestesia geral e ventilação seletiva, sendo aplicado através de pleuroscopia. Os efeitos adversos mais comuns são febre e dor torácica, durando geralmente 72 horas. $^{1}$

Uma opção terapêutica amplamente difundida é a inserção de cateteres pleurais de longa duração, como o Dreno Pigtail e os cateteres tunelizados. Estes últimos são inseridos na cavidade pleural de forma que uma pequena porção permaneça alocada no subcutâneo. ${ }^{1}$ São indicados em casos de obstrução brônquica e encarceramento neoplásico do pulmão, situações em que a pleurodese não costuma apresentar sucesso. Pleurectomia subtotal ou total e decorticação podem controlar derrames malignos refratários a outras abordagens. ${ }^{10}$

O tratamento oncológico, apesar da reduzida eficácia no controle dos sintomas, deve ser mantido. ${ }^{1}$ A quimioterapia pode apresentar resposta no linfoma, câncer de mama, câncer de próstata, câncer de ovário, carcinoma de pulmão, pequenas células e tumores de células germinativas. Radioterapia pode ser de ajuda em casos de predomínio de linfonodomegalias mediastinais (ex. linfoma). ${ }^{10}$

\section{Derrame pleural recidivante não neoplásico}

O tratamento do derrame pleural recidivante segue uma linha terapêutica semelhante ao tratamento do derrame neoplásico. Pacientes assintomáticos não necessitam abordagem. A toracocentese de repetição é apropriada para derrames que não reacumulam rapidamente. No geral, caso seja necessária mais de uma vez por mês, outras abordagens devem ser pensadas. A síndrome de reexpansão pulmonar é evitada não retirando mais que $1500 \mathrm{ml}$ de líquido pleural e suspendendo o procedimento em casos de dor torácica ou queda da pressão pleural abaixo de $-20 \mathrm{cmH}_{2} \mathrm{O} \cdot{ }^{11}$

Nos casos de rápido reacúmulo de líquido pleural, a pleurodese se torna uma boa opção terapêutica. Os cateteres tunelizados de longa duração com drenagem externa intermitente também são usados, principalmente quando a pleurodese está contraindicada. Procedimentos mais invasivos dificilmente são necessários. ${ }^{11}$

Investigation of a unilateral pleural effusion in adults: British Thoracic Society pleural disease guideline 2010. Thorax. 2010;65(Suppl 2):ii4-17.

4. Light RW. Clinical practice: pleural effusion. N Engl J Med. 2002; 346(25):1971-7.

5. Noriega G. Protocolo diagnóstico del derrame pleural. Medicine. 
2014;11(67):4035-43.

6. Heffner JE. Diagnostic thoracentesis. UpToDate. 2015 [acesso em: 2017 Jan 02]. Disponível em: www.uptodate.com/contents/ diagnostic-thoracentesis

7. Heffner JE. Diagnostic evaluation of a pleural effusion in adults: initial testing. UpToDate. 2015 [acesso em: 2017 Jan 02]. Disponível em: www.uptodate.com/contents/diagnostic-evaluation-of-a-pleuraleffusion-in-adults-initial-testing

8. Gary Lee YC. Diagnostic evaluation of pleural effusion in adults: Additional tests for undetermined etiology. UpToDate. 2015 [acesso em: 2017 Jan 02]. Disponível em: www.uptodate.com/contents/ diagnostic-evaluation-of-pleural-effusion-in-adults-additional-testsfor-undetermined-etiology
9. Stark P. Imaging of pleural effusions in adults. UpToDate. 2015 [acesso em: 2017 Jan 02]. Disponível em: www.uptodate.com/ contents/imaging-of-pleural-effusions-in-adults

10. Heffner JE. Management of malignant pleural effusions. UpToDate. 2015 [acesso em: 2017 Jan 02]. Disponível em: www. uptodate.com/contents/management-of-malignant-pleural-effusions

11. Doelken P. Management of refractory nonmalignant pleural effusions. UpToDate. 2015 [acesso em: 2017 Jan 02]. Disponível em: www.uptodate.com/contents/management-of-refractorynonmalignant-pleural-effusions

\section{Como citar:}

Dantas GC, Reis RC. Protocolo de abordagem de derrame pleural. Rev Med UFC. 2018 abr-jun;58(2):67-74. 